\title{
Prolonging the Lifetime of Two-Tiered Wireless Sensor Networks with Mobile Relays
}

\author{
Theodore C. Kotsilieris ${ }^{1}$ and George T. Karetsos ${ }^{2}$ \\ ${ }^{1}$ Department of Health \& Welfare Units Administration, Technology Education Institute of Kalamata, 24100 Kalamata, Greece \\ ${ }^{2}$ Department of Information Technology and Telecommunications, Technology Education Institute of Larissa, 41110 Larissa, Greece \\ Correspondence should be addressed to Theodore C. Kotsilieris; tkots@teikal.gr
}

Received 31 December 2012; Accepted 26 January 2013

Academic Editors: C.-Y. Chow, S. Ozdemir, and Y. Yu

Copyright ( 2013 T. C. Kotsilieris and G. T. Karetsos. This is an open access article distributed under the Creative Commons Attribution License, which permits unrestricted use, distribution, and reproduction in any medium, provided the original work is properly cited.

\begin{abstract}
We propose a clustering scheme for wireless sensor nodes in hierarchical wireless sensor networking architectures that employs mobile relay nodes in order to achieve energy conservation and network lifetime prolongation. The key aspects of our scheme are relay node relocation and reclustering when failures are detected. The performance of the proposed approach is evaluated via simulations for various topology layouts based on the sensor node population and number of mobile relay nodes employed. The results show significant energy savings in particular for topologies with large numbers of sensors.
\end{abstract}

\section{Introduction}

Wireless sensor networks are becoming more and more popular nowadays due to the significant advantages they present in carrying out specialized monitoring tasks in certain application areas. These areas vary from military [1] and target tracking [2] to healthcare [3-5] and environmental monitoring [6-8]. However, there exist certain aspects that need to be addressed during deployment in order to achieve the best possible functionality and eliminate as much as possible problems that are inherent in wireless sensors technology and networking [9].

One of the most important design issues of a wireless sensor network that should be carefully treated is energy consumption and management that directly affects the network's lifetime. Many research approaches have been proposed already as potential solutions to this problem. The suggested methods span from MAC layer algorithms [10-15] and topology control actions $[16,17]$ to hierarchical architectures that adopt the introduction of special purpose nodes, called relay nodes [18-20].

Research efforts on hierarchical architectures, also called two-tiered architectures, apart from introducing the role of a relay node $(\mathrm{RN})$ or cluster head $(\mathrm{CH})$, go further by applying clustering techniques that aim at minimizing the sensor communication cost $[18,21]$. Several techniques have been proposed in the literature that aim to avoid rapid battery drainage that consequently may cause network connectivity failures or sensing area holes [22-25]. In single-hop operation of wireless sensor nodes (WSNs), data propagation towards the destination (i.e., the base station (BS)) that will further process the sensed data is accomplished by the relay nodes. Thus, in hierarchical deployments the critical factor is the lifetime achieved by the relay nodes which has to be enhanced as much as possible. Towards this, a measurable objective is the number of data gathering rounds that can be carried out by an RN. A data gathering round of an RN corresponds to the procedure of receiving the collected data from the nodes in its cluster and transmitting them to the base station [26].

This work proposes a clustering scheme for SNs that enhances energy conservation and prolongs the network's lifetime. The main difference with other similar approaches is that the RNs are not considered stationary nodes expected to function at the locations that were initially deployed but with mobility capabilities that allow them to move to new locations designated by optimal energy consumption management procedures. In particular we extend the work presented in [18] so that the RNs can move to new locations, a feature that 
in conjunction with re-clustering can lead to energy consumption minima.

The rest of this work is organized as follows. Section 2 presents background work on the field of topology control for WSNs and clustering algorithms. In Section 3, we propose an algorithm that applies postdeployment topology control functions in an already formed cluster so that energy consumption is minimized. Simulation results that highlight the energy conservation achieved by the proposed algorithm are presented in Section 4. Finally, Section 5 concludes our work and suggests future research items.

\section{WSN Mobility-Based Topology Control and Clustering Algorithms}

With the proliferation of WSN technologies and applications, the need for incorporating mobility has been identified as a means to enhance their performance. To this end various mobility models for WSNs have been introduced that range from sensing node mobility $[20,21]$ to base station (BS) movement in predefined or autonomous paths [27-29].

In the case of mobile sensor nodes, the movement capability is limited due to energy saving constraints and usually is identified within a cluster's scope. On the contrary, a mobile base station or data collector does not present such limitations as they can be usually fed with unlimited energy. However, an energy conservation scheme based on a mobile $\mathrm{BS}$ is not appropriate for real-time data collection because a sensor that is ready to transmit should wait until it gets in the communication range of the BS. In order to confront with the implied data collection delay of this scheme, aggregation and processing of sensed data take place, which are usually performed by relay nodes [30].

2.1. Sensor Mobility Models. Sensor mobility is a recent addition to the WSNs characteristics that has been employed to face initial deployment problems and communication holes that may arise due to node failure or battery drainage. The solutions proposed vary from working with redundant nodes [31] ready to move - in a straightforward or cascaded modewhen asked so to using functional nodes of the WSN to replace failed ones or for balancing network traffic [32]. The mobile nodes of the latter case may be plain sensors with battery limitations or relays with enriched mobility and computational capabilities [33].

Our motivation of addressing such a sensor network redeployment problem combined with relay node mobility constraints is to evaluate further enhancements on scalability and power efficiency of clustering algorithms. In this paper a sensor field (topology) is divided (clustered) into groups (clusters) in accordance with the constraints defined by the Integer Linear Programming (ILP) formulas given in [18]. In this work, after the initial clustering has taken place, no further re-deployment (re-clustering) actions are taken in order to further minimize energy wastage.

Our work goes a step further towards network lifetime maximization. The RNs are not considered stationary devices designated to perform data collection and cluster coordination, but may move to new locations within the formed cluster in order to achieve power consumption savings. Also, RNs are not considered to be subject of power limitations, at least not at the same extent that SNs do. The trajectory of each RN is calculated taking into account all the necessary constraints so as not to incur any side effects to the clusters' structure.

2.2. Sensor Communication: Radio Model. The main components of a sensor node are a sensing circuit, a data processing unit, and a radio transceiver. However, the power required for communication is the dominant part of the total power consumption in a sensor network. A radio model that defines in detail the communication power consumption is outlined in [34].

As this model suggests, energy is consumed at a rate of $E_{T x \text {-elec }} 50 \mathrm{~nJ} /$ bit for running the transmitter circuitry and $E_{R x \text {-elec }} 50 \mathrm{~nJ} / \mathrm{bit}$ for the receiving one, which for the rest of this paper will be called $E_{\text {elec }}$. Additionally, the transmit amplifier also dissipates $\varepsilon_{\mathrm{amp}}$ amount of energy (a typical value is $100 \mathrm{pJ} / \mathrm{bit} / \mathrm{m}^{2}$ ) to transmit one bit of data over unit distance.

In a typical WSN, energy consumption is mainly due to three reasons: packet transmission, packet reception, and data processing.

Thus, by taking into account the energy loss per bit due to transmission at a distance $d$, the energy dissipated by the amplifier to transmit $k$ bits is given by

$$
E_{T x \text {-amp }}(k, d)=\varepsilon_{\mathrm{amp}} * k * d^{q},
$$

where $q$ corresponds to the path loss exponent. Therefore, the overall energy consumption to transmit $k$ bits is given by

$$
\begin{aligned}
E_{T x}(k, d) & =E_{T x \text {-elec }}(k)+E_{T x \text {-amp }}(k, d) \\
& \Longrightarrow E_{T x}(k, d)=E_{\text {elec }} * k+\varepsilon_{\mathrm{amp}} * k * d^{q}
\end{aligned}
$$

On the other hand, the energy dissipated to receive $k$ bits over a distance $d$ is given by

$$
E_{R x}(k)=E_{R x \text {-elec }}(k) \Longrightarrow E_{R x}(k)=E_{\text {elec }} * k .
$$

The energy consumed to process $k$ bits is defined as

$$
E_{\mathrm{CPU}}(k)=\varepsilon_{\mathrm{CPU}} * k,
$$

where $\varepsilon_{\mathrm{CPU}}(\mathrm{n} J / \mathrm{bit})$ is the energy consumed for processing a single bit.

\section{Optimal Relay Node Placement}

The clustering model proposed in [18] is derived via integerlinear-programming- (ILP-) based formulations covering not only single-hop communications, but also multihop ones, and the corresponding methods are called ILP-S and ILP-M, 
respectively. The proposed version of our algorithm performs clustering under the assumption of single-hop data transmission in order to perform a preliminary evaluation of its operation without the need to take care for intersensor communication at this stage. The main innovation introduced in our approach, called MILP-S (M stands for mobile), is the possibility to relocate the RNs since they hold mobility capabilities. The scope of their movement is to find the most suitable location within a cluster so that the overall energy consumption is minimized. In order to locate the new position of the relay node that minimizes the overall energy consumption, we consider all the locations that are not further than a distance $r_{\max }$ from each node that comprises the cluster. Distance $r_{\max }$ is the transmission range of a sensor. This constraint is necessary in order to preserve the topology of the already formulated clusters. If such a location is found, the relay node is forwarded towards it and the network lifetime is increased.

3.1. Step 1: Single-Hop ILP Cluster Formulation. The adopted network model follows a two-tiered wireless network architecture where $s$ sensor nodes form the sensing area (i.e., the lower layer), and $r$ relay nodes comprise the upper layer. The sink node (or base station) is considered to reside outside the sensing area at a long distance from both sensing and relay nodes. Thus, the total number of nodes, $N$, of the network is $s+r+1$. Each sensor is designated by label $a$, where $1 \leq a \leq s$. A relay is denoted by a label $b$, with $s+1 \leq b \leq s+r$ while the label of the base station is $s+r+1$. It should be noted that sensor and relay nodes operate in different ways. The former perform plain sensing while the latter gather the cluster's sensed data and transmit them to the sink node. Thus, energy depletion and consequent shutdown of a sensing node may affect only the accuracy of measurements. On the other hand, a possible energy depletion of a relay node, which is actually the cluster head, results in total loss of the gathered data from the respective cluster.

The simulation topology consists of a grid of sensor nodes with relay nodes placed arbitrarily on the grid and a base station. Although the sensor nodes are considered to be uniformly distributed, the relay nodes are randomly placed. This topology formation follows the algorithm's concept. The lower layer that consists of the sensing nodes that create data has little to do with the cluster formation procedure which depends on the relay nodes' characteristics such as position, number, and transmission range. In addition, our algorithm goes one step further: after the initial random cluster formation, we deal also with the issue of prolonging the network's lifetime by appropriately moving and relocating the relay nodes that are less in number and have superior processing and energy characteristics. In such a setup, each sensor can belong to only one cluster and each relay node can supervise only one cluster. ILP-S proposes a formulation that creates $r$ clusters (equal to the number of the relay nodes), each one consisting of $N_{b}$ nodes, that leads to network lifetime maximization. In the single-hop model, each relay node receives data only from sensors that belong to its cluster and directly transmits them to the corresponding base station. A cluster is defined through a binary matrix $X_{i j}$ as

$$
X_{i j}=\left\{\begin{array}{rr}
1, & \text { if node } i \text { belongs to the cluster of } \mathrm{RN} j \\
0, & \forall i, j: 1 \leq i \leq s, s+1 \leq j \leq s+r \\
\text { in any other case. }
\end{array}\right.
$$

The condition above (according to the predefined notations) can be re-written as

$$
X_{i j}= \begin{cases}1, & \text { if } n_{i, j} \in S_{j} \quad \forall i, j: 1 \leq i \leq\left|S_{j}\right| \\ 0, & \text { in any other case. }\end{cases}
$$

The algorithm's objective is to minimize the energy dissipated per round of operation, $E_{\text {single-hop }}$, subject to the following constraints

Each sensor node must join only one cluster:

$$
\sum_{j=s+1}^{s+r} \mathrm{X}_{i j}=1, \quad \forall i, 1 \leq i \leq s .
$$

A sensor node may transmit to the relay node only if the latter is located within the transmission range $r_{\max }$ of the node

$$
X_{i j} d_{i j} \leq r_{\max } \quad \forall i, 1 \leq i \leq s \forall j, s+1 \leq \mathrm{j} \leq s+r,
$$

where $d_{i, j}$ denotes the Euclidean distance between locations $i$ and $j$ (i.e., $\left.d_{i j}=\sqrt{\left(x_{i}-x_{j}\right)^{2}+\left(y_{i}-y_{j}\right)^{2}}\right)$.

The total number of bits $f_{j}$, generated in cluster $j$ and received by relay node $j$, is computed by

$$
\sum_{i=1}^{s} b_{i} X_{i j}=f_{j}, \quad \forall j \in N .
$$

Furthermore, the maximum energy spent by an $\mathrm{RN}$ in a round is computed by

$$
\begin{gathered}
E_{\text {single-hop }, j}=E_{T X \text {-elec }} f_{j}+E_{R X \text {-elec }} f_{j}+\varepsilon_{\text {amp }} f_{j} d_{j, s+r+1}^{q}, \\
E_{\text {single-hop }}=\sum_{\forall j \in N} E_{\text {single-hop }, j} .
\end{gathered}
$$

3.2. Step 2: Relay Node Movement to New Locations. We have devised a replacement algorithm that is executed to calculate the optimum location of the relay nodes as soon as the respective clusters are formed. Let $L$ denote the set of all possible locations for the placement of a sensor within the sensing area. Since every node belongs to a cluster, the following holds: $L=\left\{L_{s+1}, L_{s+2}, \ldots, L_{s+r}\right\}$, where $L_{j}, \forall j: s+1 \leq j \leq$ $s+r$ denotes all the available locations for cluster $j$ while $r$ denotes the number of relay nodes and consequently the clusters that will be formed. The possible locations that an RN may be placed are a subset of the locations included in the cluster. Let $P L_{j} \subseteq L_{j}$ be a set of feasible distinct locations (a distinct location is represented by $l_{i, j}, \forall i, j: 1 \leq i \leq\left|L_{j}\right|$, $s+1 \leq j \leq s+r)$ for the relay node $i$ according to

$$
\min \left(E_{\text {Single-hop }, j}\left(l_{i j}\right)\right) \text {, }
$$

where $l_{i, j} \in P L_{j}:\left\|l_{\text {current }}-l_{i, j}\right\| \leq r_{\max }, \forall i \in\left\{1 \cdots\left|L_{j}\right|\right\}$.

The above mentioned algorithm is described in detail in Algorithm 1. 


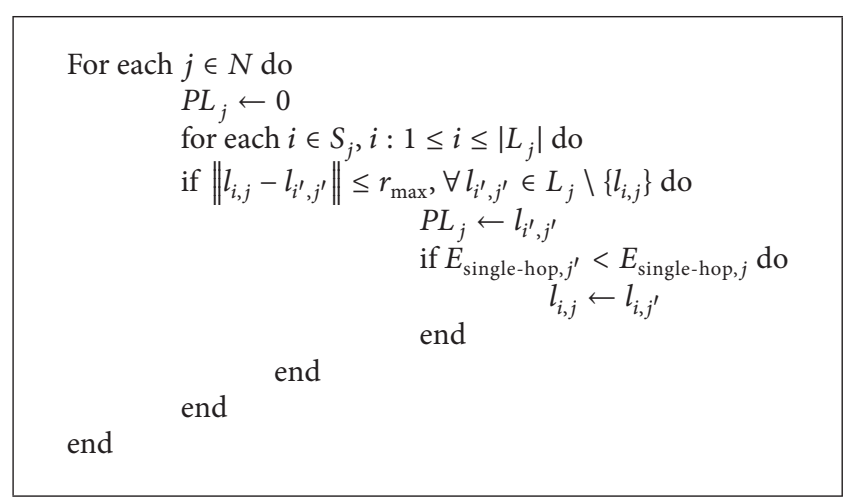

Algorithm 1: The relay node movement algorithm.

\section{Simulation Results}

4.1. Environment Setup. The communication energy consumption is calculated according to the first-order radio model [34] with the constants

$$
\begin{aligned}
& E_{T X \text {-elec }}=50 \mathrm{nj} / \mathrm{bit}, \\
& E_{R X \text {-elec }}=50 \mathrm{nj} / \mathrm{bit}, \\
& \varepsilon_{\text {amp }}=100 \mathrm{pJ} / \mathrm{bit} / \mathrm{m}^{2}, \\
& q=2, \\
& r_{\max }=200 \mathrm{~m} .
\end{aligned}
$$

Our simulation environment comprised a combination of MATLAB and FICO Xpress Optimization suite that allowed us to solve the ILP clustering problem quickly and efficiently.

The simulated topologies scaled from 576 nodes ( 24 units of length by 24 units of width) to 10.000 nodes (100 units long by 100 units wide) while the RNs populations varied from 4 to 25 . Such a dense distribution of sensing and relay nodes is allowed by the low pernode cost which supports at the same time the appropriate redundancy so as to obtain accurate field data.

The simulation results reveal an improvement to the energy consumption compared to ILP-S that increases as the network topology population grows. After executing the clustering algorithm, on a square-shaped topology of 6400 sensor nodes, uniformly distributed on an area of 80 units length to 80 units width with 4 relay nodes available, we obtain the clustering depicted in Figure 1.

Accordingly, Figure 2 presents a $100 * 100$ sensor node grid topology with 9 relay nodes.

Each cluster in Figures 1 and 2 contains a different number of nodes because the initial placement of the relay nodes within the sensing area is performed randomly. On the other hand, the distribution of the sensing nodes is done in a uniform manner due to its simplicity for grid topology formation and neutrality in producing the simulation results. It is also easier to study the algorithm's scalability performance as the sensing nodes increase. We have to note also that despite the fact that the grid sizes may seem unrealistic they are in essence very important for assessment purposes. Further simulations will be performed over special purpose topologies where harsh environments affect the sensing nodes

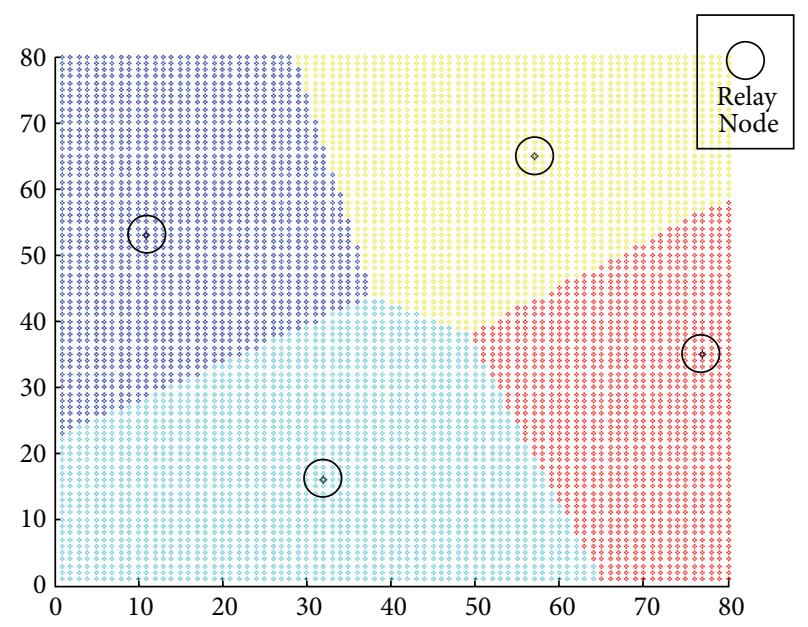

FIGURE 1: Clustering results with 4 relay nodes for an $80 * 80$ sensor node topology.

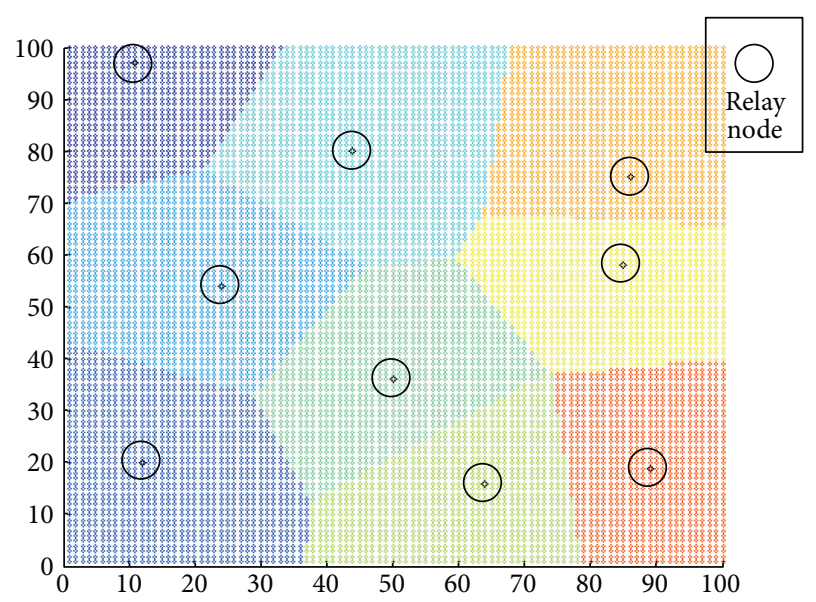

FIGURE 2: Clustering results with 9 relay nodes for a $100 * 100$ sensor node topology.

placement but mobility is a necessary feature in order to gain network connectivity.

4.2. Performance Evaluation of MILP-S over ILP-S. The performance results obtained are given in this section. Our method's primary goal is to cluster the sensor nodes around an RN such that energy consumption within the cluster is minimized. The resulting energy consumptions when forming 4, 9, 16, and 25 clusters per sensing area are depicted in Table 1 and are compared to the ILP-S method.

Table 1 presents the performance improvement of MILP$S$ over the ILP-S method versus the number of nodes for different grid formations and relay nodes. The comparison metric is energy consumption with $4,9,16$, and 25 relay nodes. Table 1 also includes the percentage of improvement of our algorithm over ILP-S. For clarity, the results provided in Table 1 are plotted in Figure 3.

As it can be easily observed, MILP-S method surpasses ILP-S in energy-saving terms. Performance improvement 
TABLE 1: Comparison of energy consumption between ILP-S and MILP-S for several grid sizes.

\begin{tabular}{|c|c|c|c|c|c|c|c|c|c|c|}
\hline \multirow{2}{*}{\multicolumn{2}{|c|}{ Energy conservation method }} & \multicolumn{9}{|c|}{ Grid size (nodes) } \\
\hline & & \multirow{2}{*}{$\begin{array}{c}24 \times 24 \\
(576) \\
2,58 \%\end{array}$} & \multirow{2}{*}{$\begin{array}{c}30 \times 30 \\
(900)\end{array}$} & \multirow{2}{*}{$\begin{array}{c}40 \times 40 \\
(1600)\end{array}$} & \multirow{2}{*}{$\begin{array}{c}50 \times 50 \\
(2500)\end{array}$} & \multirow{2}{*}{$\begin{array}{c}60 \times 60 \\
(3600)\end{array}$} & \multirow{2}{*}{$\begin{array}{c}70 \times 70 \\
(4900)\end{array}$} & \multirow{2}{*}{$\begin{array}{c}80 \times 80 \\
(6400)\end{array}$} & \multirow{2}{*}{$\begin{array}{c}90 \times 90 \\
(8100)\end{array}$} & \multirow{2}{*}{$\begin{array}{c}100 \times 100 \\
(10000)\end{array}$} \\
\hline & Performance & & & & & & & & & \\
\hline \multirow[t]{3}{*}{4} & ILP-S & 0,00785 & 0,012935 & 0,024945 & 0,043936 & 0,072339 & 0,102909 & 0,138025 & 0,209471 & 0,282038 \\
\hline & MILP-S & 0,007647 & 0,012199 & 0,023184 & 0,038871 & 0,063403 & 0,090113 & 0,119877 & 0,178073 & 0,236846 \\
\hline & Performance & $3,19 \%$ & $4,22 \%$ & $5,28 \%$ & $9,18 \%$ & $9,45 \%$ & $13,29 \%$ & $15,86 \%$ & $16,38 \%$ & $18,61 \%$ \\
\hline \multirow[t]{3}{*}{9} & ILP-S & 0,007615 & 0,012212 & 0,022697 & 0,038056 & 0,057182 & 0,086448 & 0,121281 & 0,159343 & 0,220506 \\
\hline & MILP-S & 0,007373 & 0,011697 & 0,021498 & 0,034562 & 0,051780 & 0,074958 & 0,102047 & 0,133237 & 0,179472 \\
\hline & Performance & $8,88 \%$ & $9,63 \%$ & $9,80 \%$ & $10,44 \%$ & $11,19 \%$ & $11,56 \%$ & $11,95 \%$ & $12,90 \%$ & $14,31 \%$ \\
\hline \multirow[t]{3}{*}{16} & ILP-S & 0,007578 & 0,012098 & 0,021989 & 0,035727 & 0,054401 & 0,076694 & 0,103474 & 0,138666 & 0,18038 \\
\hline & MILP-S & 0,006906 & 0,010933 & 0,019834 & 0,031996 & 0,048315 & 0,067825 & 0,091112 & 0,120772 & 0,154576 \\
\hline & Performance & $7,42 \%$ & $8,39 \%$ & $9,56 \%$ & $10,29 \%$ & $11,49 \%$ & $12,38 \%$ & $12,94 \%$ & $13,11 \%$ & $15,88 \%$ \\
\hline \multirow[t]{2}{*}{25} & ILP-S & 0,007537 & 0,011954 & 0,021917 & 0,035073 & 0,052546 & 0,073813 & 0,100676 & 0,131826 & 0,168469 \\
\hline & MILP-S & 0,006978 & 0,010951 & 0,019821 & 0,031463 & 0,046508 & 0,064677 & 0,087652 & 0,114541 & 0,141715 \\
\hline
\end{tabular}

TABLE 2: Network lifetimes for ILP-S and MILP-S for various network topologies.

\begin{tabular}{|c|c|c|c|c|c|c|c|c|c|c|}
\hline \multirow{2}{*}{\multicolumn{2}{|c|}{ Energy conservation method }} & \multicolumn{9}{|c|}{ Grid size (nodes) } \\
\hline & & $\begin{array}{c}24 \times 24 \\
(576)\end{array}$ & $\begin{array}{c}30 \times 30 \\
(900)\end{array}$ & $\begin{array}{c}40 \times 40 \\
(1600)\end{array}$ & \multirow{2}{*}{$\begin{array}{c}50 \times 50 \\
(2500)\end{array}$} & \multirow{2}{*}{$\begin{array}{c}60 \times 60 \\
(3600) \\
14,09 \%\end{array}$} & \multirow{2}{*}{$\begin{array}{c}70 \times 70 \\
(4900)\end{array}$} & \multirow{2}{*}{$\begin{array}{c}\begin{array}{c}80 \times 80 \\
(6400)\end{array} \\
15,14 \%\end{array}$} & \multirow{2}{*}{$\begin{array}{c}90 \times 90 \\
(8100)\end{array}$} & \multirow{2}{*}{$\begin{array}{c}100 \times 100 \\
(10000)\end{array}$} \\
\hline & & $2,65 \%$ & $6,03 \%$ & $7,60 \%$ & & & & & & \\
\hline \multirow[t]{3}{*}{4} & ILP-S & 637 & 387 & 200 & 114 & 69 & 49 & 36 & 24 & 18 \\
\hline & MILP-S & 654 & 410 & 216 & 129 & 79 & 55 & 42 & 28 & 21 \\
\hline & & $3,29 \%$ & $4,40 \%$ & $5,58 \%$ & $10,11 \%$ & $10,43 \%$ & $15,33 \%$ & $18,85 \%$ & $19,59 \%$ & $22,86 \%$ \\
\hline \multirow[t]{3}{*}{9} & ILP-S & 657 & 409 & 220 & 131 & 87 & 58 & 41 & 31 & 23 \\
\hline & MILP-S & 678 & 427 & 233 & 145 & 97 & 67 & 49 & 38 & 28 \\
\hline & & $9,74 \%$ & $10,65 \%$ & $10,87 \%$ & $11,66 \%$ & $12,60 \%$ & $13,08 \%$ & $13,57 \%$ & $14,82 \%$ & $16,69 \%$ \\
\hline \multirow[t]{3}{*}{16} & ILP-S & 660 & 413 & 227 & 140 & 92 & 65 & 48 & 36 & 28 \\
\hline & MILP-S & 724 & 457 & 252 & 156 & 103 & 74 & 55 & 41 & 32 \\
\hline & & $8,02 \%$ & $9,16 \%$ & $10,58 \%$ & $11,47 \%$ & $12,98 \%$ & $14,13 \%$ & $14,86 \%$ & $15,09 \%$ & $18,88 \%$ \\
\hline \multirow[t]{2}{*}{25} & ILP-S & 663 & 418 & 228 & 143 & 95 & 68 & 50 & 38 & 30 \\
\hline & MILP-S & 717 & 457 & 252 & 159 & 108 & 77 & 57 & 44 & 35 \\
\hline
\end{tabular}

varies from $4 \%$, for sensing fields that contain approximately 500 sensors, to $15 \%-20 \%$ for large topologies with 10.000 nodes. Accordingly the achieved lifetimes are also improved (see Table 2). The measurements regarding network lifetimes were conducted with an initial energy of 5 Joules per SN.

Applying the power-law least-squares method to the computed network lifetimes results in a fit, depicted in Figure 3, with the coefficient of determination, $R^{2}$, varying from 0.9978 to 0.9997 (see Table 3). Thus, the lifetime of such a network layout can be predicted through an equation of the form $y=A x^{B}$. The values of $A$ and $B$ coefficients are assumed to be related to the parameters used during the ILP formulation of the clustering problem and more specifically to the dataflow from each sensor (i.e., bits per node), the relay node location, and the first-order radio constants (i.e., $E_{T X \text {-elec }}$, $\left.E_{R X \text {-elec }}, \varepsilon_{\text {amp }}, r_{\max }, q\right)$.

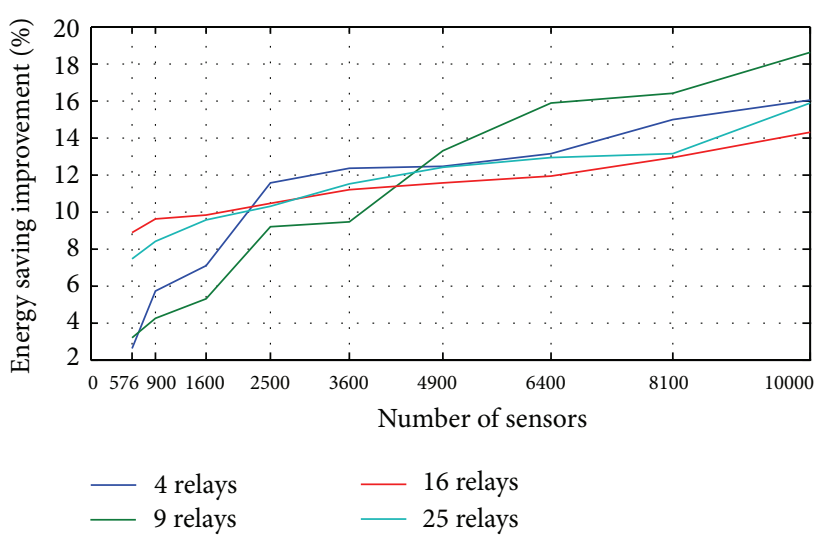

FIGURE 3: Energy saving improvement of MILP-S over ILP-S clustering method for several grid sizes. 
TABLE 3: The $R^{2}$ coefficient of determination for various relays.

\begin{tabular}{lc}
\hline Number of relays & $R^{2}$ \\
\hline 4 & 0,9978 \\
9 & 0,9989 \\
16 & 0,9995 \\
25 & 0,9997 \\
\hline
\end{tabular}

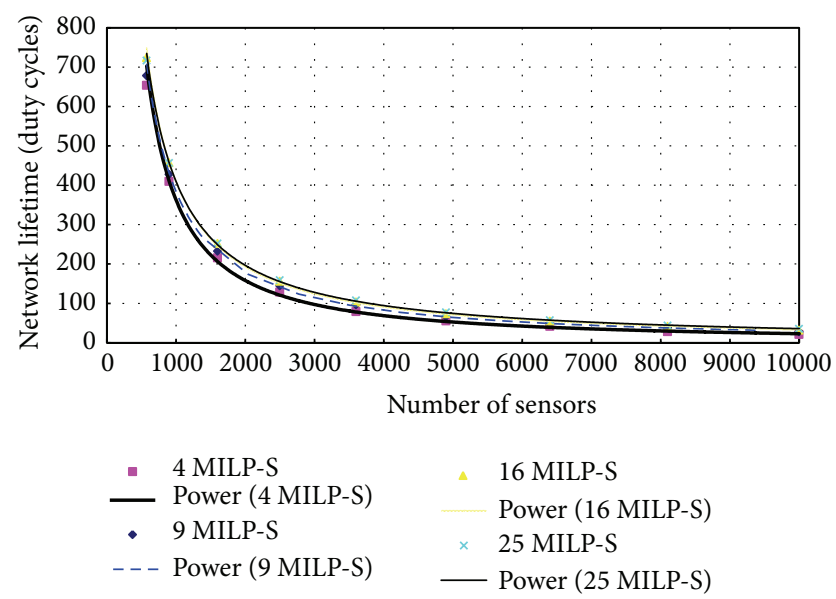

Figure 4: Power-law least square fitting to the simulation results of the MILP-S method.

From Figure 4, we observe that the network lifetime decreases at unacceptable levels when the number of sensors exceeds 2000 nodes. This result has to be taken into account during deployments with such or higher number of sensors.

\section{Conclusions}

In this work a clustering method for prolonging the lifetime of two-tiered wireless sensor networks is proposed and evaluated. In particular, we extend static clustering through the provision of mobility for the corresponding relay nodes. The obtained simulation results show a significant performance improvement on energy savings when the relay nodes are allowed to move into new locations within the initially formulated clusters. This performance improvement may exceed 20\% when large topologies with thousands of sensors are considered. Furthermore, a curve fitting analysis on the results revealed that the network lifetime follows a powerlaw distribution. Finally, future work will focus on optimizing network coverage, connectivity, and performance in case of sensor or even relay node failures in special purpose topologies.

\section{References}

[1] C. Schurgers and M. B. Srivastava, "Energy efficient routing in wireless sensor networks," in Proceedings of the IEEE Military Communications Conference, Communications for NetworkCentric Operations: Creating the Information Force (MILCOM '01), vol. 1, pp. 357-361, 2001.
[2] X. Wang, J. J. Ma, S. Wang, and D. W. Bi, "Cluster-based dynamic energy management for collaborative target tracking in wireless sensor networks," Sensors, vol. 7, no. 7, pp. 1193-1215, 2007.

[3] S. Jiang, Y. Cao, S. Iyengar et al., "CareNet: an integrated wireless sensor networking environment for remote healthcare," in Proceedings of the ICST 3rd International Conference on Body Area Networks (ICST '08), pp. 9:1-9:3, Brussels, Belgium, 2008.

[4] H. Alemdar and C. Ersoy, "Wireless sensor networks for healthcare: a survey," Computer Networks, vol. 54, no. 15, pp. 2688-2710, 2010.

[5] S.-L. Chen, H. Y. Lee, C. A. Chen, H. Y. Huang, and C. H. Luo, "Wireless body sensor network with adaptive low-power design for biometrics and healthcare applications," IEEE Systems Journal, vol. 3, no. 4, pp. 398-409, 2009.

[6] P. Corke, T. Wark, R. Jurdak, W. Hu, P. Valencia, and D. Moore, "Environmental wireless sensor networks," Proceedings of the IEEE, vol. 98, no. 11, pp. 1903-1917, 2010.

[7] H. Liu, Z. Meng, and M. Wang, "A wireless sensor network for cropland environmental monitoring," in International Conference on Networks Security, Wireless Communications and Trusted Computing (NSWCTC '09), pp. 65-68.

[8] F. Ingelrest, G. Barrenetxea, G. Schaefer, M. Vetterli, O. Couach, and M. Parlange, "SensorScope: application-specific sensor network for environmental monitoring," ACM Transactions on Sensor Networks, vol. 6, no. 2, pp. 1-32, 2010.

[9] I. Akyildiz, "Wireless sensor networks: a survey," Computer Networks, vol. 38, pp. 393-422, 2002.

[10] J.-H. Chang and L. Tassiulas, "Maximum lifetime routing in wireless sensor networks," IEEE/ACM Transactions on Networking, vol. 12, no. 4, pp. 609-619, 2004.

[11] H. Wang, N. Agoulmine, M. Ma, and Y. Jin, "Network lifetime optimization in wireless sensor networks," IEEE Journal on Selected Areas in Communications, vol. 28, no. 7, pp. 1127-1137, 2010.

[12] R. Braynard, A. Silberstein, and C. Ellis, "Extending network lifetime using an automatically tuned energy-aware MAC protocol," Lecture Notes in Computer Science, vol. 3868, pp. 244259, 2006.

[13] X. Yang, T. Camp, H. Yang, and A. P. Petropulu, "Extending network lifetime for ALLIANCES," Computer Communications, vol. 32, no. 17, pp. 1837-1851, 2009.

[14] R. A. Rashid, W. M. A. E. W. Embong, A. Zaharim, and N. Fisal, "Development of energy aware TDMA-based MAC protocol for wireless sensor network system," European Journal of Scientific Research, vol. 30, no. 4, pp. 571-578, 2009.

[15] F. Naït-Abdesselam, B. Bensaou, T. Soëte, and K. L. Hung, "OMAC: an organized energy-aware MAC protocol for wireless sensor networks," in Proceedings of the IEEE International Conference on Communications (ICC '07), pp. 3648-3653, June 2007.

[16] P. Santi, "Topology control in wireless ad hoc and sensor networks," ACM Computing Surveys, vol. 37, no. 2, pp. 164-194, 2005.

[17] Y. Wang, "Topology control for wireless sensor networks," in Wireless Sensor Networks and Applications, Y. Li, M. T. Thai, and W. Wu, Eds., pp. 113-147., Springer, Boston, Mass, USA, 2008.

[18] A. Bari, A. Jaekel, and S. Bandyopadhyay, "Clustering strategies for improving the lifetime of two-tiered sensor networks," Computer Communications, vol. 31, no. 14, pp. 3451-3459, 2008.

[19] O. Younis, M. Krunz, and S. Ramasubramanian, "Node clustering in wireless sensor networks: recent developments and deployment challenges," IEEE Network, vol. 20, no. 3, pp. 2025,2006 . 
[20] A. A. Abbasi and M. Younis, "A survey on clustering algorithms for wireless sensor networks," Computer Communications, vol. 30, no. 14-15, pp. 2826-2841, 2007.

[21] L. S. Jayashree, S. Arumugam, and N. Rajathi, "E2LBC: an energy efficient load balanced clustering technique for heterogeneous wireless sensor networks," in IEEE IFIP International Conference on Wireless and Optical Communications Networks, pp. 1-7, 2006.

[22] N. Dimokas, D. Katsaros, and Y. Manolopoulos, "Energyefficient distributed clustering in wireless sensor networks," Journal of Parallel and Distributed Computing, vol. 70, no. 4, pp. 371-383, 2010.

[23] M. K. Watfa, O. Mirza, and J. Kawtharani, "BARC: a Battery Aware Reliable Clustering algorithm for sensor networks," Journal of Network and Computer Applications, vol. 32, no. 6, pp. 1183-1193, 2009.

[24] Y.-Y. W. Jie Jia, J. Chen, G. R. Chang, and Y. Y. Wen, "Efficient cover set selection in wireless sensor networks," Acta Automatica Sinica, vol. 34, no. 9, pp. 1157-1162, 2008.

[25] W. B. Heinzelman, A. P. Chandrakasan, and H. Balakrishnan, "An application-specific protocol architecture for wireless microsensor networks," IEEE Transactions on Wireless Communications, vol. 1, no. 4, pp. 660-670, 2002.

[26] K. Kalpakis, K. Dasgupta, and P. Namjoshi, "Maximum lifetime data gathering and aggregation in wireless sensor networks," in Proceedings of the Joint International Conference on Wireless LANs and Home Networks (ICWLHN '02) and Networking (ICN '02), pp. 685-696, 2002.

[27] Y. Yang, M. I. Fonoage, and M. Cardei, "Improving network lifetime with mobile wireless sensor networks," Computer Communications, vol. 33, no. 4, pp. 409-419, 2010.

[28] J. Rao and S. Biswas, "Network-assisted sink navigation for distributed data gathering: stability and delay-energy tradeoffs," Computer Communications, vol. 33, no. 2, pp. 160-175, 2010.

[29] W. Alsalih, H. Hassanein, and S. Akl, "Placement of multiple mobile data collectors in wireless sensor networks," Ad Hoc Networks, vol. 8, no. 4, pp. 378-390, 2010.

[30] A. A. Somasundara, A. Kansal, D. D. Jea, D. Estrin, and M. B. Srivastava, "Controllably mobile infrastructure for low energy embedded networks," IEEE Transactions on Mobile Computing, vol. 5, no. 8, pp. 958-972, 2006.

[31] H. Li, S. Liu, and B. Hu, "Research on node sleep/wake-up mechanism in WSN based on energy threshold setting," in Proceedings of the 5th International Conference on Wireless Communications, Networking and Mobile Computing (WiCOM '09), pp. 1-4, September 2009.

[32] C. Y. Chang and H. R. Chang, "Energy-aware node placement, topology control and MAC scheduling for wireless sensor networks," Computer Networks, vol. 52, no. 11, pp. 2189-2204, 2008.

[33] T. Banerjee, B. Xie, J. H. Jun, and D. P. Agrawal, "Increasing lifetime of wireless sensor networks using controllable mobile cluster heads," Wireless Communications and Mobile Computing, vol. 10, no. 3, pp. 313-336, 2010.

[34] W.R. Heinzelman, A. Chandrakasan, and H. Balakrishnan, "Energy-efficient communication protocol for wireless microsensor networks," in Proceedings of the 33rd Annual Hawaii International Conference on System Sciences, January 2000. 

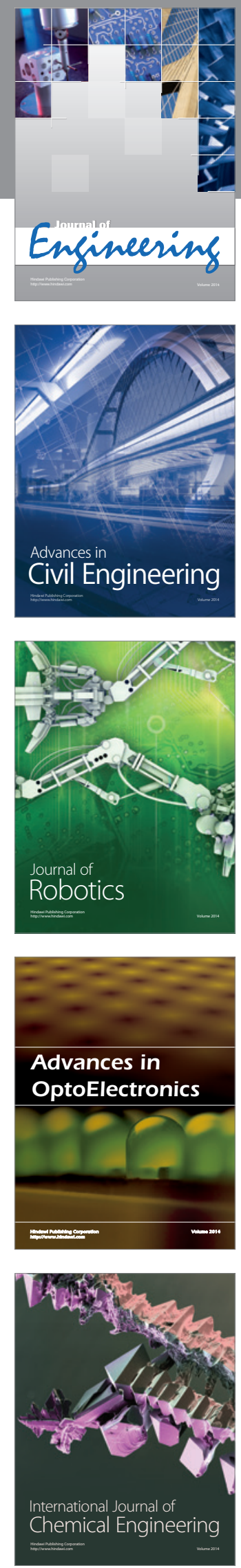

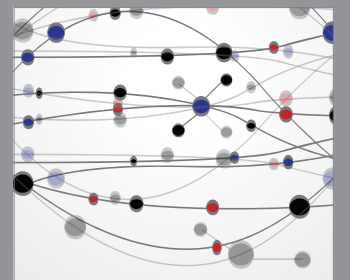

The Scientific World Journal
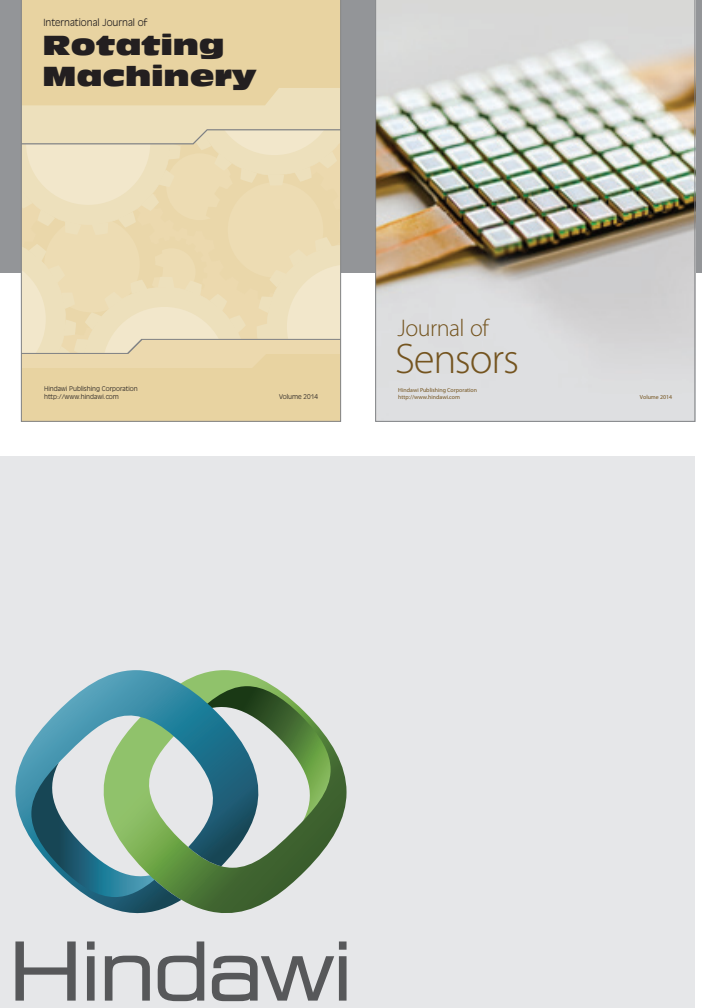

Submit your manuscripts at http://www.hindawi.com
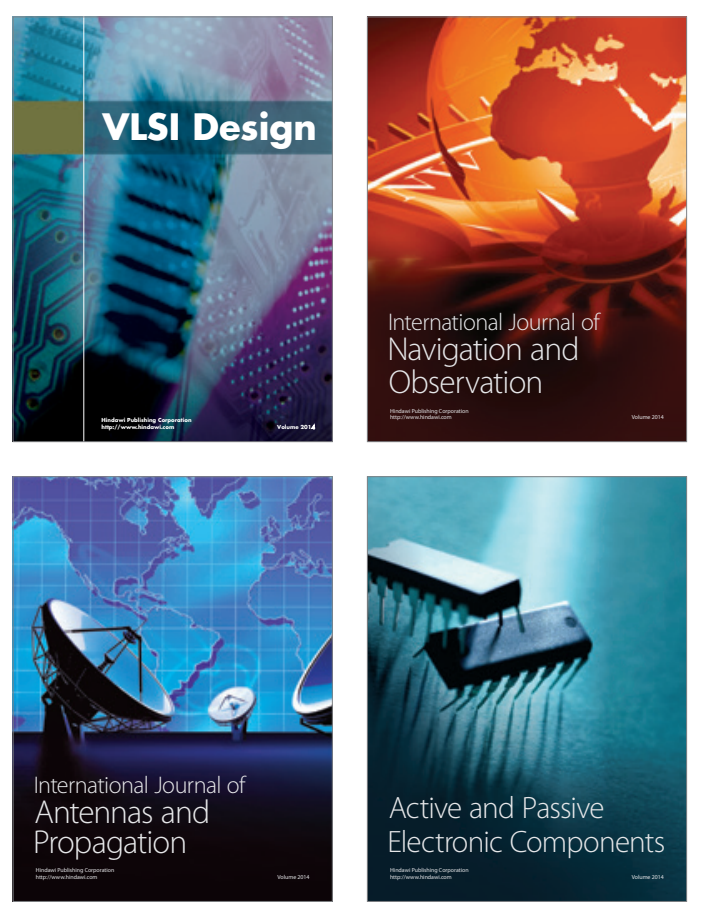
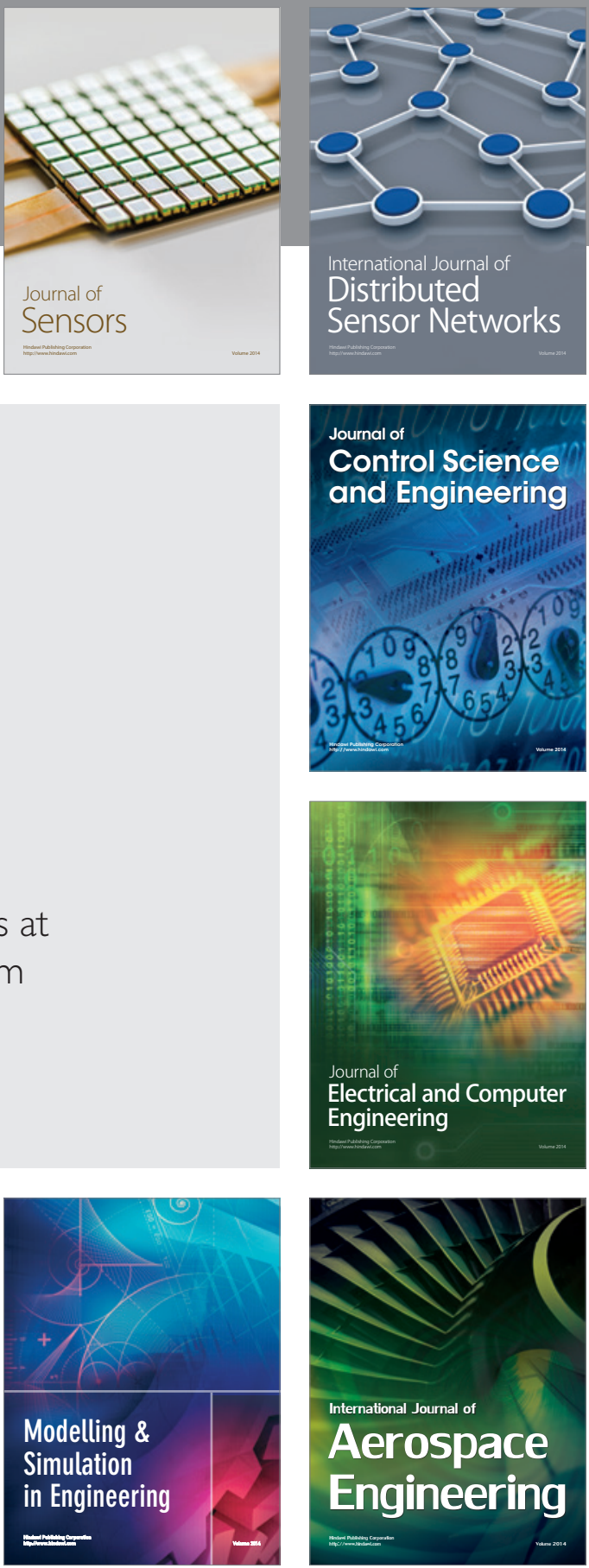

Journal of

Control Science

and Engineering
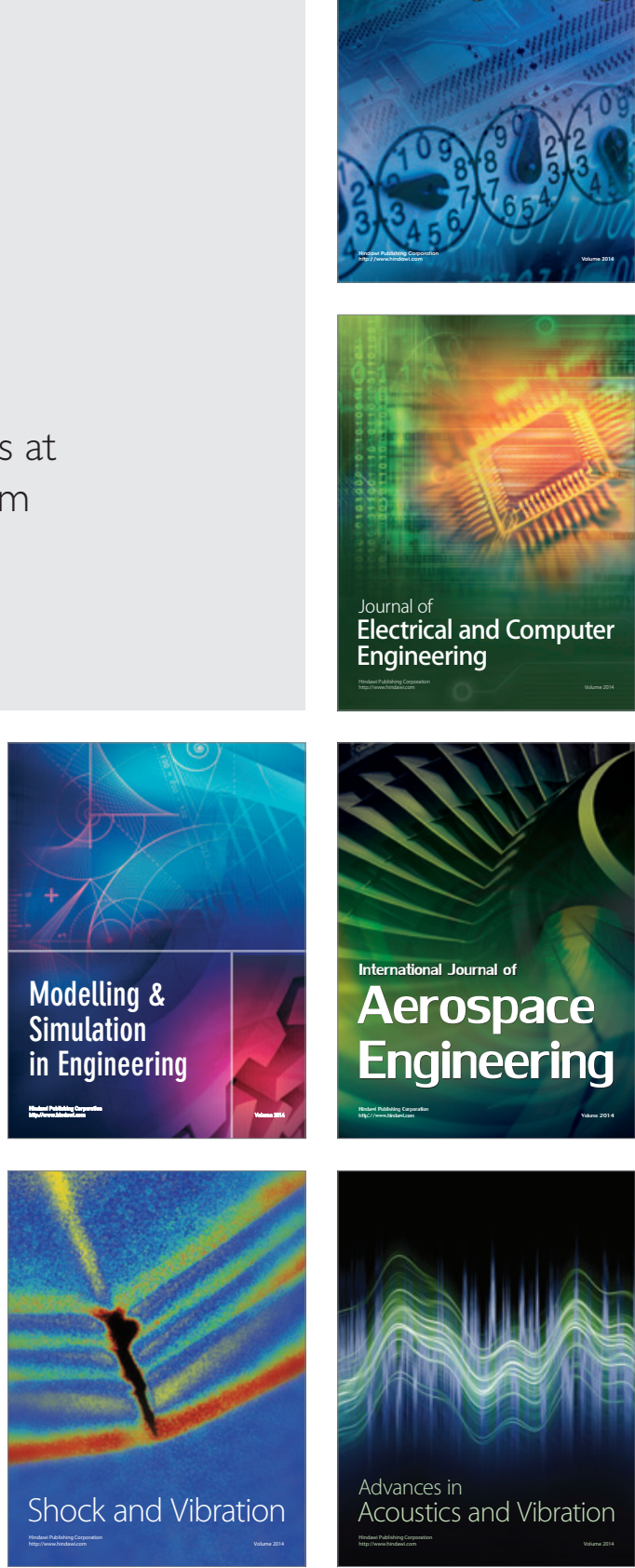